\title{
Estudo da liga alumínio-zinco como filtração alternativa em aparelhos de raios $\mathrm{X}$ odontológicos
}

\section{Study of the aluminum-zinc alloy as an alternative means of filtration in dental $X$-ray devices}

\author{
Claudia Fátima Silva HAITER* \\ Frab Norberto BÓSCOLO** \\ Francisco HAITER NETO*** \\ Solange Maria de ALMEIDA****
}

\begin{abstract}
HAITER, C. F. S.; BÓSCOLO, F. N.; HAITER NETO, F.; ALMEIDA, S. M. Estudo da liga alumínio-zinco como filtração alternativa em aparelhos de raios X odontológicos. Pesqui Odontol Bras, v. 16, n. 1, p. 77-82, jan./mar. 2002.

O objetivo neste trabalho foi avaliar os efeitos da filtração alternativa da liga alumínio-zinco em aparelhos de raios $\mathrm{X}$ odontológicos, em relação ao espectro de energia dos feixes de raios X, a taxa de kerma no ar, o índice de contraste nas radiografias obtidas e a qualidade das imagens radiográficas. Este estudo foi realizado comparando-se o filtro convencional de alumínio com várias espessuras do filtro de liga alumínio-zinco, em diferentes porcentagens do elemento zinco na liga ( $2 \%, 3 \%, 4 \%$ e $5 \%$ ). Foi utilizado um aparelho de raios X odontológico convencional, operando com $60 \mathrm{kVp} \mathrm{e}$ $70 \mathrm{kVp}$, e filmes radiográficos dos grupos D e E de sensibilidade. Foram realizadas radiografias de um "phantom" e de uma escala de densidade de alumínio. Os resultados mostraram que o uso dos filtros de liga Al- $\mathrm{Zn}$ resultou em um não-endurecimento dos feixes. A taxa de kerma no ar diminuiu em até 18,53\%, sem alteração no contraste das radiografias e a qualidade das imagens teve uma boa aceitação pelos examinadores. A análise dos resultados nos levou a concluir que o filtro de liga Al-Zn 2\%, com 1,99 mm de espessura, tensão de $60 \mathrm{kVp}$, quando usado com filme D, foi o que obteve melhor desempenho, reduzindo a taxa de kerma no ar sem variar o indice de contraste das radiografias, mantendo a qualidade da imagem aceitável para diagnóstico, sem contudo aumentar o tempo de exposição.
\end{abstract}

UNITERMOS: Raios X; Espectrometria; Ligas dentárias; Aluminio; Filtros; Filtração.

\section{INTRODUÇÃO}

O principal objetivo da Radiologia é produzir imagens radiográficas de qualidade, enquanto a exposição à radiação, para o paciente, é mantida a mais baixa possivel, sendo que o uso de filtros é um meio de se atingir esse objetivo.

A função dos filtros é de barrar principalmente os fótons de baixa energia, ou seja, aqueles que têm um maior comprimento de onda, e conseqüentemente um menor poder de penetração na matéria. Os fótons de baixa energia pouco interferem na formação da imagem, pois a sensibilidade dos filmes radiográficos usados em Odontologia é maior entre 35 e $55 \mathrm{keV}$, ou seja, em energias de médios valores. Os fótons de energia alta são responsáveis pelo aumento da radiação secundária e reduzem o contraste da imagem. Um filtro ideal deveria ser capaz de remover os fótons de baixa e alta energia do feixe, permitindo a passagem apenas dos fótons que são úteis na formação da imagem.

A adição ou substituição de filtros provoca alterações na energia média do feixe, na taxa de kerma no ar e na qualidade das imagens radiográficas.

Vários elementos foram testados com o intuito de se encontrar um filtro alternativo ao filtro convencional de alumínio, ou mesmo melhorar a sua performance. Propusemo-nos a estudar o uso do filtro de liga alumínio-zinco, em aparelhos de raios $\mathrm{X}$ odontológicos convencionais, no que se refere ao comportamento do espectro de energia, a taxa de kerma no ar, e também a qualidade das imagens radiográficas.

\section{MATERIAIS E MÉTODOS}

Primeiramente foram confeccionados filtros de uma liga monofásica, em alumínio e zinco, em dife-

\footnotetext{
*Mestre em Radiologia Odontológica; **Professor Titular; ***Professor Associado; ****Professora Assistente Doutora - Disciplina de
} Radiologia da Faculdade de Odontologia de Piracicaba da Universidade Estadual de Campinas. 
HAITER, C. F. S.; BÓSCOLO, F. N.; HAITER NETO, F.; ALMEIDA, S. M. de. Estudo da liga alumínio-zinco como filtração alternativa em aparelhos de raios X odontológicos. Pesqui Odontol Bras, v. 16, n. 1, p. 77-82, jan./mar. 2002.

rentes espessuras (aproximadamente 0,5; 1 e $2 \mathrm{~mm}$ de espessura), e com diferentes porcentagens de $\mathrm{Zn}$ na liga ( $2 \%, 3 \%$, 4\% e 5\%). O aparelho de raios $\mathrm{X}$ empregado foi da marca General Electric (General Electric Company, Milwalkee, Wisconsin, EUA), modelo GE 1000, operando com $60 \mathrm{kVp}$ e $70 \mathrm{kVp}, 10 \mathrm{~mA}$ e distância foco-filme de $40 \mathrm{~cm}$. Os filtros da liga Al-Zn foram utilizados para se obter as radiografias de uma escala de densidade de alumínio, com 8 degraus, segundo recomendações propostas por MANSON-HING; BLOXOM ${ }^{10}$ (1985). Para a execução dessas radiografias, foram utilizados filmes intrabucais fabricados pela Kodak (Eastman Kodak Company, Rochester, EUA), pertencentes aos grupos D e E de sensibilidade.

Todas as radiografias foram processadas automaticamente em uma processadora Gendex GXP (Dentsplay International, Gendex Dental X-ray Division, Des Plaines, Ilinois, EUA), utilizando-se soluções de processamento Kodak, e com tempo total de processamento de 5 minutos.

Para cada tipo de filme (D e E), foram utilizadas as tensões de 60 e $70 \mathrm{kVp}$, formando assim 4 conjuntos (D 60, D 70, E 60, E 70). Para cada conjunto de filme e $\mathrm{kVp}$, realizou-se uma radiografia da escala de densidade variando os filtros de aluminio (padrão) e Al-Zn, nas diferentes espessuras utilizadas.

Um plano piloto foi desenvolvido para determinar o tempo de exposição necessário para se obter uma radiografia com densidade satisfatória. Foram feitas radiografias da escala de densidade com tempos variando de 0,4 a 1,5 segundo, com o objetivo de se obter o tempo de exposição ideal para cada conjunto filtro/espessura do filtro $/ \mathrm{kVp} /$ tipo de filme. Para isso, essas radiografias foram submetidas a uma leitura densitométrica dos degraus dessa escala, feitas com um fotodensitômetro digital da marca MRA. (Indústria de Equipamentos MRA. Ltda, Ribeirão Preto - SP). O tempo de exposição ideal para cada conjunto foi determinado quando o degrau de espessura de $10 \mathrm{~mm}$ da escala apresentava densidade óptica variando de 0,9 a 1,1, de acordo com MAURIELLO et al. ${ }^{11}$ (1987).

Para a avaliação subjetiva da imagem radiográfica, foi confeccionado um "phantom" a partir de um segmento de mandíbula do lado esquerdo, incluída em resina acrílica, permitindo a simulação de tecidos moles. As radiografias desse "phantom" foram feitas utilizando-se os diferentes tipos de filtros/espessura do filtro $/ \mathrm{kVp} /$ tipo de filme e com os tempos de exposições previamente determina- dos para cada conjunto. A avaliação subjetiva foi feita em uma sala com redução da luz ambiente, apenas com a luz do negatoscópio. Por uma avaliação conjunta de 3 radiologistas, foram conferidas notas a essas radiografias, que variavam de 1 (inaceitável) a 4 (ótima). De posse dos valores de cada degrau da escala de densidade, também foi calculado o contraste relativo ou indice de contraste das radiografias.

Foram feitas as medidas da camada semi-redutora (CSR) pelo método de interposição de filtros de alumínio, e da taxa de kerma no ar, com uma câmara de ionização Victoreen 06-526 (Radcal Corporation, EUA). A medição do espectro do feixe de raios $\mathrm{X}$ foi feita com um detector de telureto de cádmio e zinco, com um sistema especial de colimação, adaptado por BECKER ${ }^{1}$ em 1997.

\section{RESULTADOS}

\section{Espectro de energia do feixe de raios $X$}

Com relação ao espectro de energia dos feixes filtrados pela liga $\mathrm{Al}-\mathrm{Zn}$, pudemos encontrar 4 diferentes tipos de comportamento, que foram:

1. feixes com menor quantidade de fótons, porém com energia média igual ao do feixe filtrado por alumínio (ex.: Al-Zn 2\% com 1,99 mm de espessura e Al-Zn 5\% com 2,08 mm de espessura, ambos com tensão de $60 \mathrm{kVp}$, e Al-Zn 3\% com $1,98 \mathrm{~mm}$; Al-Zn $4 \%$ com 1,98 mm e Al-Zn 5\% com 2,08 mm de espessura, todos com tensão de $70 \mathrm{kVp}$ );

2. feixes com quantidade de fótons e energia média igual ao do feixe filtrado por aluminio (ex.: Al-Zn $2 \%$ com $1,13 \mathrm{~mm}$ de espessura e as tensões de 60 e $70 \mathrm{kVp}$ );

3. feixes com maior quantidade de fótons e energia média igual ao do feixe filtrado por alumínio (ex.: Al-Zn 3\% com 0,42 mm e Al-Zn 4\% com $0,94 \mathrm{~mm}$ de espessura, ambos com tensão de $70 \mathrm{kVp}$ );

4. feixes com maior quantidade de fótons e energia média menor que a do feixe filtrado por aluminio (ex.: Al-Zn $2 \%$ com $0,46 \mathrm{~mm}$ de espessura, nas tensões de 60 e $70 \mathrm{kVp}$ ).

Os Gráficos 1, 2, 3 e 4 exemplificam os quatro tipos de comportamentos obtidos, respectivamente.

Devemos destacar que não ocorreu em nossos resultados o endurecimento do feixe, ou seja, aumento da energia média do feixe decorrente da utilização dos filtros de liga Al-Zn. 
HAITER, C. F. S.; BÓSCOLO, F. N.; HAITER NETO, F.; ALMEIDA, S. M. de. Estudo da liga alumínio-zinco como filtração alternativa em aparelhos de raios X odontológicos. Pesqui Odontol Bras, v. 16, n. 1, p. 77-82, jan./mar. 2002.

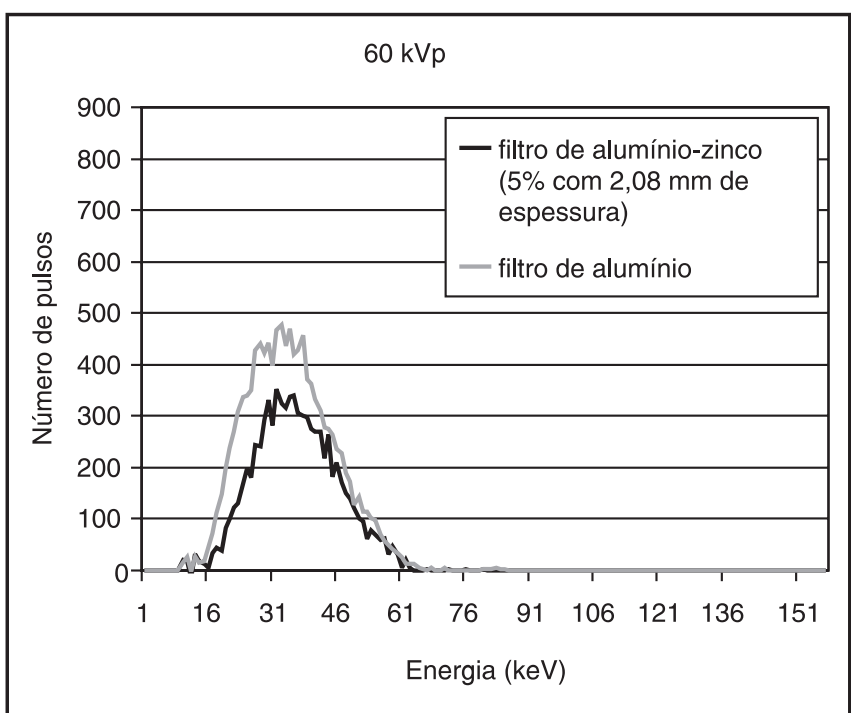

GRÁFICO 1 - Feixe filtrado pela liga Al-Zn apresenta menor quantidade de fótons, porém com energia média igual à do feixe filtrado por alumínio.

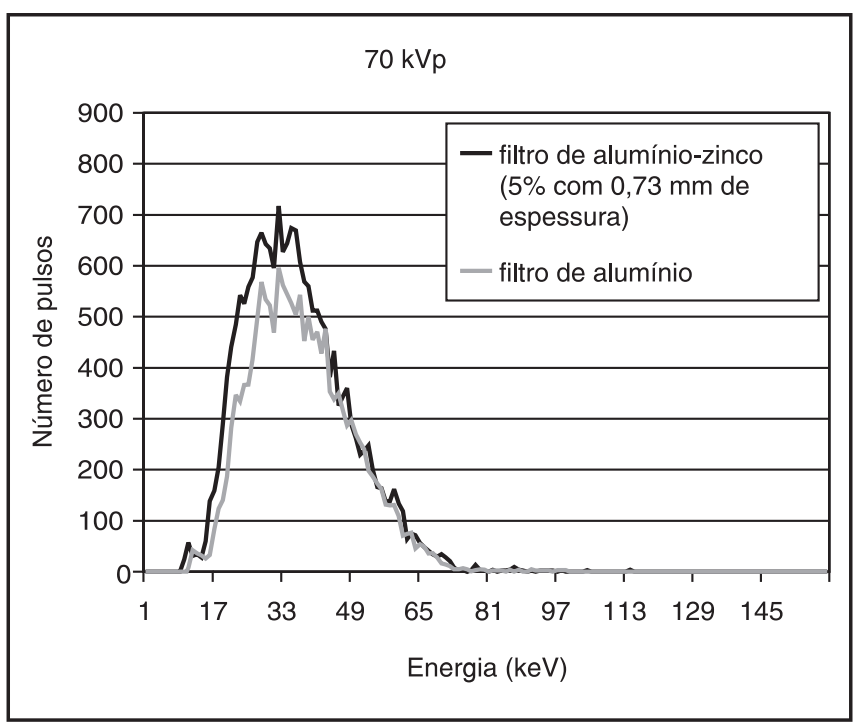

GRÁFICO 3 - Feixe filtrado pela liga Al-Zn apresenta maior quantidade de fótons, porém com energia média igual à do feixe filtrado por alumínio.

\section{Taxa de kerma no ar}

A porcentagem de aumento ou diminuição na taxa de kerma foi obtida comparando-se os valores de kerma no ar medidos quando se utilizou o filtro padrão de alumínio e os filtros da liga Al-Zn, nos tempos necessários para se obter radiografias com densidades dentro de uma escala aceitável.

As taxas de kerma no ar produzidas pelos filtros de liga $\mathrm{Al}-\mathrm{Zn}$ apresentaram redução com a utilização de Al-Zn 5\%, 2,08 mm de espessura, filme D e

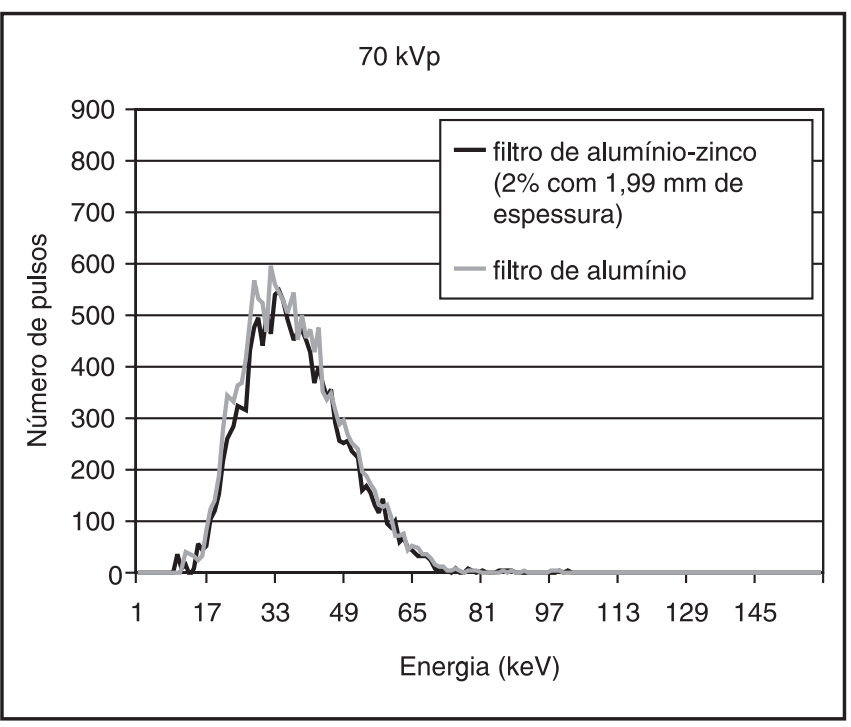

GRÁFICO 2 - Feixe filtrado pela liga Al-Zn apresenta quantidade de fótons e energia média iguais às do feixe filtrado por alumínio.

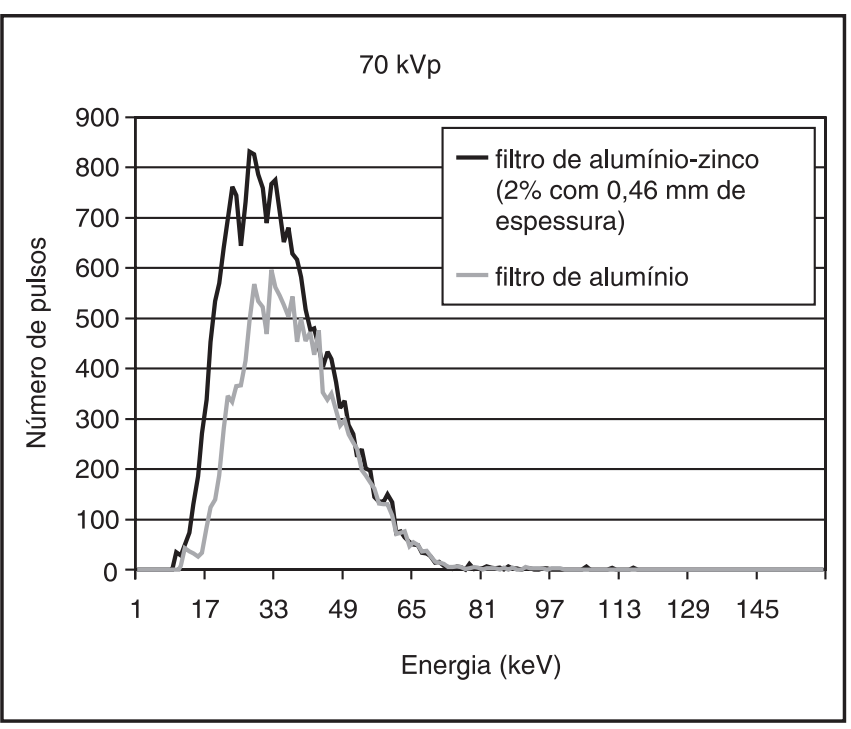

GRÁFICO 4 - Feixe filtrado pela liga Al-Zn apresenta maior quantidade de fótons e energia média menor do que a do feixe filtrado por aluminio.

tensões de 60 e $70 \mathrm{kVp}$, porém com aumento no tempo de exposição. Também ocorreu redução da taxa de kerma com o filtro Al-Zn 2\%, 1,99 mm de espessura, filme D e $60 \mathrm{kVp}$, sem, entretanto, neste caso, ocorrer aumento no tempo de exposição. Essas reduções foram de 0,45\%, 18,53\% e $11,49 \%$, respectivamente.

\section{Contraste}

O contraste das radiografias obtidas com os filtros da liga manteve-se praticamente constante, 
HAITER, C. F. S.; BÓSCOLO, F. N.; HAITER NETO, F.; ALMEIDA, S. M. de. Estudo da liga alumínio-zinco como filtração alternativa em aparelhos de raios X odontológicos. Pesqui Odontol Bras, v. 16, n. 1, p. 77-82, jan./mar. 2002.

apresentando uma variação máxima de 13\% em relação ao contraste das radiografias obtidas com filtro de alumínio, sendo que as radiografias que apresentaram os melhores índices de contraste foram as obtidas com os seguintes filtros da liga Al-Zn: $2 \%$ com $1,99 \mathrm{~mm}$ e $5 \%$ com $2,08 \mathrm{~mm}$ de espessura, ambos com filme D e tensão de $60 \mathrm{kVp}$; $3 \%$ com $1,95 \mathrm{~mm}, 4 \%$ com $1,98 \mathrm{~mm}$ e $5 \%$ com $2,08 \mathrm{~mm}$ de espessura, com tensão de $70 \mathrm{kVp}$ e os filmes D e E.

\section{Qualidade da imagem radiográfica}

Em nossos resultados, não ocorreu perda na qualidade das imagens radiográficas decorrente do uso dos filtros Al-Zn, pois pudemos observar que as radiografias obtidas com os filtros $\mathrm{Al}-\mathrm{Zn}$ apresentaram uma boa aceitação pelos examinadores.

\section{DISCUSSÃO}

RICHARDS ${ }^{15}$ (1964) observou que a remoção de fótons de energia mais alta pelo filtro pode acarretar a necessidade de um aumento no tempo de exposição a fim de compensar essa perda de fótons úteis na formação da imagem. Em nossa pesquisa, esse fato também ocorreu, uma vez que, para os filtros da liga que apresentaram um poder de filtração maior que o do alumínio, o tempo de exposição necessário para se obter uma radiografia de boa densidade foi de $25 \%$ a $50 \%$ maior que o utilizado para o filtro de alumínio convencional. Os nossos resultados também indicaram que a energia média dos feixes filtrados com a liga $\mathrm{Al}-\mathrm{Zn}$, nas porcentagens e espessuras que apresentaram maior filtração, sem aumentar o endurecimento do feixe, encontravam-se dentro da escala de 30 a $40 \mathrm{keV}$, resultados esses também encontrados por GONÇALVES $^{6}$ (2000), que utilizou filtros de liga AlCu.

Os estudos de CHO et al. ${ }^{3}$, em 1978, utilizando a filtração adicional de cobre e alumínio, de CHAKERA et al. $^{2}$, em 1982, que usaram filtração adicional de érbio e alumínio, e de KAPA et $a l .^{8}$ (1990), que utilizaram filtros de metais terras raras mostraram uma alteração na qualidade do feixe com endurecimento do mesmo em relação ao filtro padrão de alumínio. Em nosso trabalho, em nenhum momento observamos o endurecimento do feixe, independentemente da porcentagem de zinco na liga, da espessura e da tensão utilizada. Devemos aqui ressaltar que os trabalhos acima apresentados utilizaram filtração adicional ao filtro convencional de alumínio, e nós substituímos a filtração convencional de alumínio por filtros de uma liga Al-Zn. Nossos resultados concordam com os de CHO et al. ${ }^{3}$ (1978), em que o aumento do $\mathrm{kVp}$ não alterou as energias médias dos feixes.

Com relação à taxa de kerma no ar, os trabalhos de RICHARDS ${ }^{16}$ (1970), GELSKEY; BAKER ${ }^{5}$ (1981) e KOHN et al. ${ }^{9}$ (1988) demonstraram que a colocação de filtros adicionais ao filtro convencional de alumínio provocou uma diminuição da dose de radiação, porém com um aumento no tempo de exposição de 40 a $127 \%$. Em nossos resultados, os filtros de liga Al-Zn que tiveram redução da taxa de kerma também tiveram um aumento no tempo de exposição de até $50 \%$, com exceção do filtro Al-Zn $2 \%$ com $1,99 \mathrm{~mm}$, a $60 \mathrm{kVp}$ e filme $\mathrm{D}$, para o qual o tempo de exposição permaneceu constante, o que mostrou ser esse um filtro capaz de reduzir a exposição à radiação, sem comprometer a carga do tubo. A maior porcentagem de redução da taxa de kerma no ar encontrada em nosso trabalho foi de $18,53 \%$, e a menor foi de $0,45 \%$. GONÇALVES ${ }^{6}$ (2000), utilizando filtros de liga $\mathrm{AlCu}$, encontrou redução da taxa de kerma no ar de até 47,33\%, e PARDINI $^{14}$ (1995) encontrou redução da dose de $75 \%$ com o uso de filtros de zinco.

Estudando o contraste obtido nas radiografias produzidas com a utilização de filtros da liga Al-Zn, pudemos notar que, de um modo geral, este foi mantido bastante próximo ao índice de contraste das radiografias produzidas com filtros convencionais de alumínio, sendo que a variação máxima foi de $13 \%$. De acordo com o estudo de OISHI; PARFITT $^{13}$, em 1976, quando ocorreu um aumento na filtração do feixe, o contraste das radiografias aumentou. Devemos ressaltar, porém, que o autor utilizou apenas filtros de alumínio, em diferentes espessuras, daí talvez proceda a discordância de nossos resultados com os encontrados pelos autores. MAURIELLO et al. ${ }^{11}$, em 1987, encontraram uma diminuição mínima e não clinicamente significante nos indices de contraste de radiografias obtidas com filtros terras raras.

Em nosso trabalho, pudemos observar que, para o filtro da liga Al-Zn $2 \%$ com 1,99 mm de espessura a $60 \mathrm{kVp}$ e no tempo de exposição usado para filme $\mathrm{D}$, ocorreu uma redução na taxa de kerma, juntamente com a manutenção do índice de contraste e sem ocasionar um aumento na carga do tubo. Resultados diferentes foram apresentados por FARMAN et $a l^{4}$, em 1989, e por JANGLAND; AXELSSON ${ }^{7}$, em 1990, que relataram uma 
HAITER, C. F. S.; BÓSCOLO, F. N.; HAITER NETO, F.; ALMEIDA, S. M. de. Estudo da liga alumínio-zinco como filtração alternativa em aparelhos de raios X odontológicos. Pesqui Odontol Bras, v. 16, n. 1, p. 77-82, jan./mar. 2002.

diminuição da dose aliada a uma perda de contraste e aumento da carga do tubo quando estudaram filtros adicionais de ítrio e nióbio.

A qualidade da imagem radiográfica foi estudada por meio de uma avaliação subjetiva em que foi possivel observar que as radiografias que obtiveram uma melhor classificação foram, em sua maioria, as que utilizaram filme D. Esses dados coincidem com os do trabalho de MAURIELLO et al. ${ }^{12}$ (1989) e de WHITE; GRATT ${ }^{18}$ (1991), que relataram que as radiografias produzidas com o filtro testado e filme D tiveram qualidade para diagnóstico comparável às radiografias produzidas com filtro convencional de aluminio.

Em nosso trabalho, quando ocorreu uma dimi- nuição na taxa de kerma no ar, a qualidade das imagens se mantiveram constantes. O mesmo resultado foi obtido por STIRLING; HOLLOWAY ${ }^{17}$ (1990) em que os autores concluíram que a filtração adicional de nióbio reduziu a dose mas não influenciou a qualidade das radiografias.

\section{CONCLUSÃO}

As ligas de $\mathrm{Al}-\mathrm{Zn}$ nas percentagens de $2 \%$ com $1,99 \mathrm{~mm}$ e $5 \%$ com $2,08 \mathrm{~mm}$ para $60 \mathrm{kVp}$; e $3 \%$ com $1,95 \mathrm{~mm}, 4 \%$ com $1,98 \mathrm{~mm}$ e $5 \%$ com $2,08 \mathrm{~mm}$ para $70 \mathrm{kVp}$ podem ser uma opção para a substituição do filtro convencional em aparelhos odontológicos.

HAITER, C. F. S.; BÓSCOLO, F. N.; HAITER NETO, F.; ALMEIDA, S. M. de. Study of the aluminum-zinc alloy as an alternative means of filtration in dental X-ray devices. Pesqui Odontol Bras, v. 16, n. 1, p. 77-82, jan./mar. 2002.

The aim of this study was to evaluate the effects of alternative filtration with the aluminum-zinc alloy in dental X-ray devices, regarding the energy spectrum, dose, contrast and image quality. This study was carried out comparing the conventional aluminum filter with aluminum-zinc alloy filters of various thicknesses and with different percentages of zinc in the alloy $(2,3,4$ and 5\%). A conventional X-ray device operating with 60 and $70 \mathrm{kVp}$ as well as D- and E-speed films were utilized in order to obtain images of a phantom and an aluminum step wedge. The utilization of Al-Zn filters resulted in a spectrum without hardening of beams. The rate of kerma in the air decreased $18.53 \%$, without any alteration in contrast and image quality. The results revealed that the $2 \% \mathrm{Al}-\mathrm{Zn}$ alloy filter with $1.99 \mathrm{~mm}$ of thickness, utilized with the tension of $60 \mathrm{kVp}$ and D-speed film, had the best performance. It reduced the rate of kerma without varying the indices of contrast and maintained image quality at acceptable levels, without increasing the time of exposure.

UNITERMS: X rays; Spectrometry; Dental alloys; Aluminum; Filters; Filtration.

\section{REFERÊNCIAS BIBLIOGRÁFICAS}

1. BECKER, P. H. B. Espectrometria de feixes de raios $\mathbf{X}$ com detector de telureto de cádmio e zinco. 1997. 98 p. Dissertação (Mestrado em Ciências em Engenharia Nuclear) - Instituto Militar de Engenharia do Rio de Janeiro, Ministério do Exército, Secretaria de Ciência e Tecnologia.

2. CHAKERA, T. M. H.; FLEAY, R. F.; HENSON, P. W. et al. Doses reduction in radiology using heavy metal foils. $\mathbf{B r}$ J Radiol, v. 55, n. 659, p. 853-858, Nov. 1982.

3. CHO, T.; UEHARA, S.; YOSHIMURA, A. et al. Dental X-ray spectrometry with a $\mathrm{Si}(\mathrm{Li})$ semiconductor. Oral Surg, v. 45, n. 6, p. 965-977, June 1978.

4. FARMAN, A. G.; PEREZ, C.; JACOBSON, A. et al. Evaluation of aluminum-yttrium filtration for intraoral radiography. Oral Surg, v. 67, n. 2, p. 224-226, Feb. 1989.

5. GELSKEY, D. E.; BAKER, C. G. Energy selective filtration of dental X-ray beams. Oral Surg, v. 52, n. 5, p. 565-567, Nov. 1981.

6. GONÇALVES, A. Estudo da liga de aluminio-cobre, como filtração alternativa para radiação X. 2000. 117 p. Dissertação (Doutorado em Radiologia Odontológica) Faculdade de Odontologia de Piracicaba, Universidade de Campinas.

7. JANGLAND, L.; AXELSSON, B. Niobium filters for dose re- duction in pediatric radiology. Acta Radiol, v. 31, n. 5, p. 540-541, Sept. 1990.

8. KAPA, S. F.; TYNDALL, D. A.; OUELLETTE, T. E. The application of added beam filtration to intra-oral radiography. Dentomaxillofac Radiol, v. 19, n. 2, p. 67-74, May 1990.

9. KOHN, M. L.; GOOCH, A. W.; KELLER, W. S. Filters for radiation reduction: a comparison. Radiol, v. 167, n. 1, p. 255-257, Apr. 1988.

10. MANSON-HING, L. R.; BLOXOM, R. M. A stepwedge quality assurance test for machine and processor in dental radiography. J Am Dent Assoc, v. 110, n. 6, p. 910-913, June 1985

11. MAURIELLO, S. M.; WASHBURN, O. B.; MATTESON, S. R. Effects of rare-earth filters on patient exposure and image contrast. J Dent Res, v. 66, n. 8, p. 1326-1330, Aug. 1987.

12. MAURIELLO, S. M.; MATTESON S. R.; TYNDALL, D. A. et al. Clinical evaluation of a samarium/aluminum compound filter. Oral Surg, v. 68, n. 1, p. 108-114, July 1989.

13. OISHI, T. T.; PARFITT, G. J. Effects of varying peak kilovoltage and filtration on diagnostic dental radiographs. J Can Dent Assoc, v. 42, n. 9, p. 449-452, Sep. 1976. 
HAITER, C. F. S.; BÓSCOLO, F. N.; HAITER NETO, F.; ALMEIDA, S. M. de. Estudo da liga alumínio-zinco como filtração alternativa em aparelhos de raios X odontológicos. Pesqui Odontol Bras, v. 16, n. 1, p. 77-82, jan./mar. 2002.

14. PARDINI, L. C. Filtração da radiação $\mathbf{X}$ em Odontologia. Estudo comparativo entre filtros de aluminio, zinco e estanho. 1995. 142 p. Tese (Livre-Docência em Ciências - Departamento de Estomatologia) - Faculdade de Odontologia de Ribeirão Preto, Universidade de São Paulo, Ribeirão Preto.

15. RICHARDS, A. G. Quality of an X-ray beam. Oral Surg, v. 17, n. 6 , p. 739-744, June 1964

16. RICHARDS, A. G.; BARBOR, G. L.; BADER, J. D. et al. Sa- marium filters for dental radiography. Oral Surg, v. 29, n. 5, p. 704-715, May 1970.

17. STIRLING, G. C.; HOLLOWAY, A. F. Niobium filter versus an aluminum filter. Ont Dent, v. 67, n. 6, p. 25-30, Oct. 1990.

18. WHITE, S. C.; GRATT, B. M. Evaluation of Niobi-X filtration in intraoral radiology. Oral Surg, v. 72, n. 6, p. 746-755, Dec. 1991.

Recebido para publicação em 16/03/01 Enviado para reformulação em 25/06/01

Aceito para publicação em 05/10/01

\section{CD "18 anos de História - Memória Científica da SBPqO"}

\section{Encontra-se à disposição de todos os associados o CD "18 anos de História - Memória Científica da Sociedade Brasileira de Pesquisa Odontológica - 1984/2001".}

Informações: Secretaria da

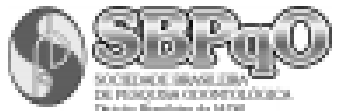

Tel.: (0**11) 3091-7855 e-mail: sbpqo@sbpqo.org.br

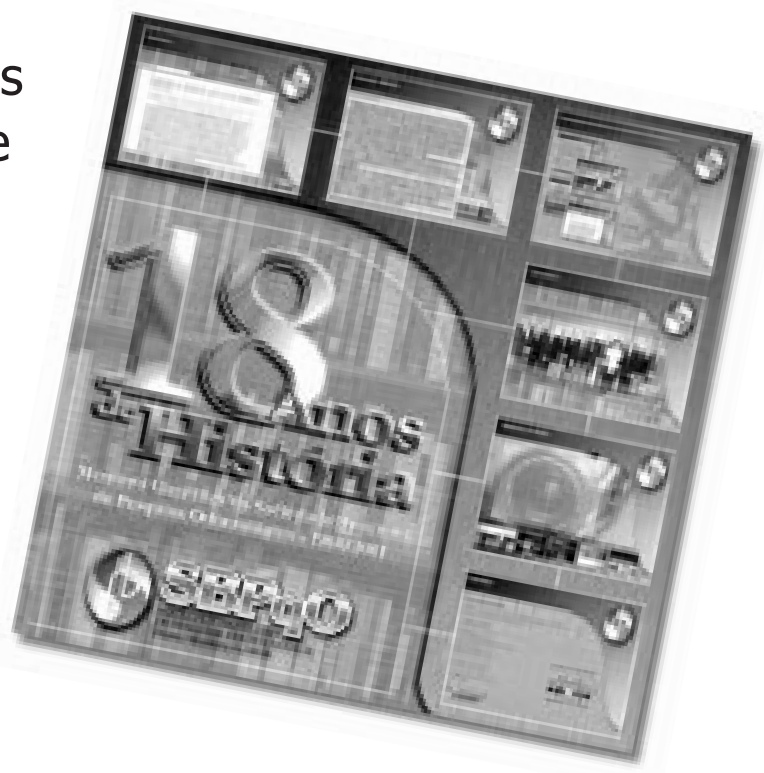

